\title{
EBM-Modell
}

\section{Netzärzte vereinfachen Honorarsystem}

\author{
Wie sieht ein Honorarsystem aus, wenn Netzärzte die Gelegenheit ha- \\ ben, selbst Hand anzulegen? Beim BMC-Kongress vom 24. bis 25. Janu- \\ ar 2017 in Berlin wurde jetzt der Schleier gelüftet: Es wird viel einfacher.
}

ndividueller KV-Fallwert plus $10 \%$ als Fallpauschale, Leistungskontrolle durch enge Kooperation im Netz: Auf Vereinfachung der Abrechnung setzen die Ärzte des Medizinischen Qualitätsnetzes Kinzigtal (MQNK) mit ihrem selbst entwickelten Honorarsystem. Es soll am 1. Januar 2018 anlaufen, berichtete Dr. Helmut Hildebrandt, Geschäftsführer von Gesundes Kinzigtal, auf dem BMC-Kongress in Berlin. Das Ergebnis des Projekts war bundesweit mit Spannung erwartet worden, weil erstmals Ärzte der Basis eine Alternative zu den etablierten Honorarsystemen entwickelt

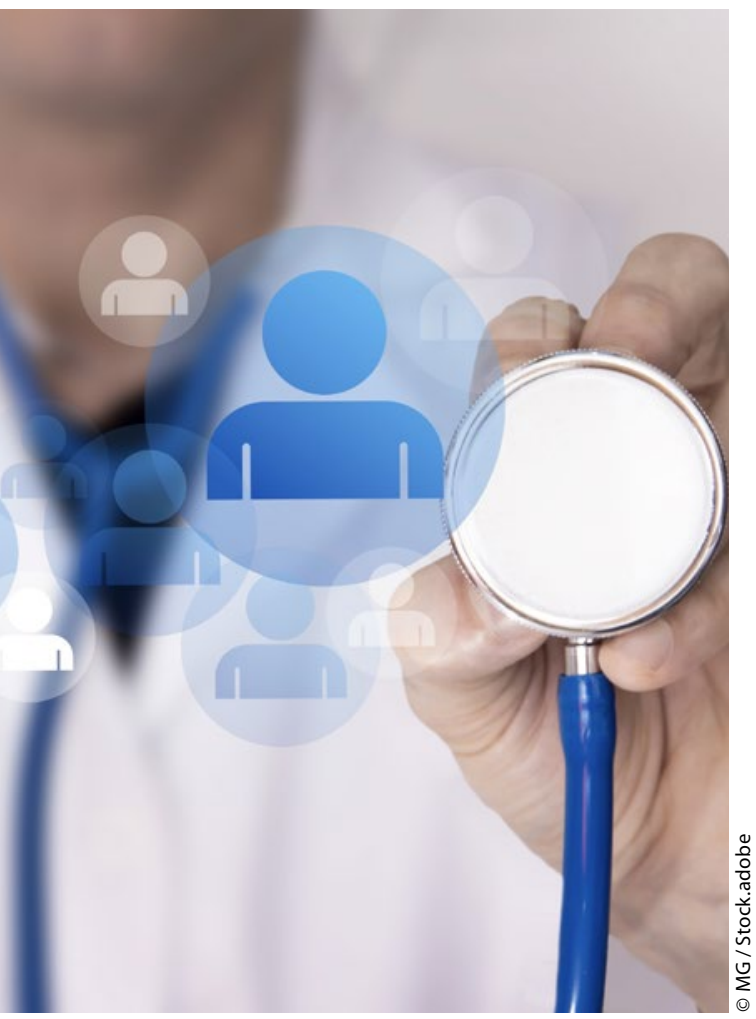

haben, wenn auch nur für den begrenzten Bereich des Kinzigtals im Schwarzwald. Gerade in Baden-Württemberg konkurrieren mit der Regelversorgung nach dem EBM und dem stärker an Pauschalen orientierten Hausarztvertragssystem bereits zwei Honorarmodelle intensiv miteinander.

Das Arztnetz im Kinzigtal ist mit der Hamburger OptiMedis AG zusammen Gesellschafter der Gesundes Kinzigtal $\mathrm{GmbH}$. Diese hat bereits vor Jahren Vollversorgungsverträge mit der $\mathrm{AOK} \mathrm{Ba-}$ den-Württemberg und der LKK abgeschlossen, über die teilnehmende Ärzte unter anderem für zusätzlichen Aufwand bei der Patientenversorgung eine Add-on-Vergütung bekommen. Außerdem gibt es eine erfolgsorientierte Vergütung, die sich daran orientiert, wie sich die Gesundheitsausgaben für die Versicherten der Krankenkassen im Vergleich zu den Morbi-RSA-Zuweisungen entwickeln. Das Netz hat in den vergangenen Jahren dabei immer einen Überschuss erwirtschaftet und konnte damit seine Aufwendungen für die Gesundheitsverbesserung der Versicherten gut tragen. Die AOK habe dann dem Netz das Angebot gemacht, ein eigenes Vergütungsmodell für ärztliche Leistungen zu entwickeln und zu erproben.

\section{Komplizierte Bereinigung}

Ein wirklich faires Vergütungssystem für alle haus- und fachärztlichen Leistungen zu entwickeln, das die Kollegen für ihr Engagement bei der Gesundheitsverbesserung der Versicherten richtig belohne, wäre so "grausam kompliziert“, dass sich Ärzte und Managementgesellschaft für einen radikal anderen Weg entschieden, berichtete Hildebrandt.
Das Honorarsystem für die errechnete Honorarsumme von letztlich 2,9 Millionen Euro sehe nun eine „vollständige Vereinfachung " vor: Für jedes neue Quartal wird für jeden Arzt ein durchschnittlicher KV-Fallwert aus den letzten vier Quartalen errechnet. Dieser Durchschnittswert wird dem Arzt garantiert und um $10 \%$ erhöht - er bildet den arztindividuellen Kinzigtal-Fallwert. Die $10 \%$ Erhöhung und zusätzliche Vergütungen für die gezielten Präventionsleistungen und die Mehrzeit finanziert die Gesundes Kinzigtal $\mathrm{GmbH}$ aus der von den Krankenkassen ausgeschütteten Erfolgsbeteiligung. „Jeder erste Kontakt mit einem Patienten im neuen Quartal von Arzt oder nicht ärztlicher Praxisassistentin löst den individuellen Fallwert aus", so Hildebrandt. Bei Vertretungsfällen gebe es $50 \%$ des Fallwerts. Vergütungen aus IV-Verträgen kämen noch hinzu. Eine Fallzahlbegrenzung entfalle. Steigerungen des Honorars der Regelversorgung werden über die Methode mit berücksichtigt. Die Dokumentation der Behandlung erfolgt wie gewohnt - die Ärzte müssten keine neuen Ziffern lernen.

\section{Transparenz durch zentrale Patientenakte}

„Ein solches Modell basiert ganz auf Vertrauen, und das ist nur innerhalb eines Arztnetzes möglich“, so Hildebrandt. Transparenz darüber, dass die Ärzte das System nicht ausnutzen und die vereinbarten Versorgungsstandards wahren, schaffe die gemeinsam genutzte zentrale Patientenakte. Außerdem gebe es auch Transparenz durch Auswertungen von Kennzahlen im Controlling - netzweit und für einzelne Praxen. Dazu gehört einmal jährlich ein individuelles Gespräch über die Entwicklung der Praxis und ihrer Kennzahlen im Vergleich zu den Netzkennzahlen, zum Beispiel Fallzahl, Klinikeinweisungen oder Verordnungskennzahlen.

Hauke Gerlof 